\title{
Transient hygrothermoelastic analysis of layered plates with one-dimensional temperature and moisture variations through the thickness
}

\author{
Ryoichi Chiba ${ }^{\mathrm{a}, *}$ \\ Yoshihiro Sugano ${ }^{\mathrm{b}}$ \\ ${ }^{a}$ Department of mechanical systems engineering, Asahikawa National College of \\ Technology, 2-2-1-6 Shunkodai, Asahikawa 071-8142, Japan \\ Phone: +81-166-55-8003 \\ Fax: +81-166-55-8003 \\ E-mail: chiba@ asahikawa-nct.ac.jp \\ ${ }^{\mathrm{b}}$ Department of mechanical systems engineering, Iwate University, 4-3-5 Ueda, \\ Morioka 020-8551, Japan \\ *Corresponding author (R. Chiba)
}

\begin{abstract}
Transient heat and moisture diffusion and the resulting hygrothermal stress field are analysed in a layered plate subjected to hygrothermal loadings at the external surfaces. The one-dimensional transient diffusion is formulated as a one-way coupled problem wherein moisture-induced effects on heat diffusion are neglected, but the exact continuity in moisture flux at layer interfaces holds unlike existing analytical studies. An analytical solution to the diffusion problem is obtained by extending a previously derived solution for double-layered plates. Hygrothermal stresses are evaluated by superposition of stresses due to the applied temperature and moisture fields. First, numerical calculations are performed for a double-layered plate to investigate the influence of moisture-flux continuity at the layer interface on hygrothermal stress distribution. Second, the obtained solutions are applied to the hygrothermoelastic problem of a functionally graded material-like (FGM-like) non-homogeneous plate whose physical properties vary along the thickness direction. Numerical results show that the use of inappropriately simplified continuity conditions for moisture flux may cause a significant error in evaluating the transient hygrothermal stresses in a layered body. Moreover, it is demonstrated that a gradual change in the material composition of FGM-like non-homogeneous plates induces considerable hygrothermal stress relaxation.
\end{abstract}


Keywords: hygrothermoelasticity, layered body, non-homogeneous material, heat and moisture diffusion, mathematical modelling, analytical solution

\section{Nomenclature}

$a$ : interface location, $\mathrm{m}$

$B$ : Biot number for heat transfer $\left(=h l / \lambda_{\text {ref }}\right)$

$B^{*}:$ Biot number for moisture transfer

$$
\left(=\chi l / \Lambda_{\text {ref }}\right)
$$

$c$ : moisture capacity, $\mathrm{kg} /\left(\mathrm{kg} \cdot{ }^{\circ} \mathrm{M}\right)$

E: Young's modulus, $\mathrm{Pa}$

$h$ : heat transfer coefficient, $\mathrm{W} /\left(\mathrm{m}^{2} \cdot \mathrm{K}\right)$

$l$ : total thickness, $\mathrm{m}$

$L:$ Luikov number $\left(=\eta / \kappa_{\text {ref }}\right)$

$m:$ moisture content $(=c \cdot u)$, wt. $\%$

$n$ : number of layers

$N$ : material gradation index

$P:$ Possnov number $\left(=\varepsilon\left(T_{\mathrm{ref}}-T_{0}\right) /\left(u_{0}-u_{\mathrm{ref}}\right)\right)$

$t$ : time, $\mathrm{s}$

$T$ : temperature, $\mathrm{K}$

$\bar{T}$ : dimensionless temperature

$$
\left(=\left(T-T_{0}\right) /\left(T_{\text {ref }}-T_{0}\right)\right)
$$

$V$ : volume fraction of a constituent material

$u$ : moisture potential, ${ }^{\circ} \mathrm{M}$

$\bar{u}$ : dimensionless moisture potential

$$
\left(=\left(u_{0}-u\right) /\left(u_{0}-u_{\mathrm{ref}}\right)\right)
$$

$x, y, z:$ coordinates, $\mathrm{m}$

$Z$ : dimensionless coordinate $(=z / l)$

$\alpha$. coefficient of thermal expansion, $\mathrm{K}^{-1}$

$\beta$ : coefficient of moisture expansion,

$$
\mathrm{cm} /\left(\mathrm{cm} \cdot \% \mathrm{H}_{2} \mathrm{O}\right)
$$

$\chi$. moisture transfer coefficient, $\mathrm{kg} /\left(\mathrm{m}^{2} \cdot \mathrm{s} \cdot{ }^{\circ} \mathrm{M}\right)$

$\delta_{i, j}$ : Kronecker delta

$\varepsilon$. thermogradient coefficient, ${ }^{\circ} \mathrm{M} / \mathrm{K}$

$\gamma$. eigenvalue for temperature field

$\eta$ : moisture diffusivity, $\mathrm{m}^{2} / \mathrm{s}$

$\kappa$. thermal diffusivity, $\mathrm{m}^{2} / \mathrm{s}$

$\bar{\kappa}$ : dimensionless thermal diffusivity

$$
\left(=\kappa / \kappa_{\text {ref }}\right)
$$

$\lambda$ : thermal conductivity, $\mathrm{W} /(\mathrm{m} \cdot \mathrm{K})$

$\mu$ : eigenvalue for moisture field

v: Poisson's ratio

$\sigma_{x x(y y)}:$ stress component

$\bar{\sigma}_{x x(y y)}:$ dimensionless stress component

$$
\left(=\sigma_{x x(y y)} /\left(E_{\text {ref }} \alpha_{\text {ref }}\left(T_{\text {ref }}-T_{0}\right)\right)\right)
$$

$\tau$. Fourier number $\left(=\kappa_{\text {ref }} t / l^{2}\right)$

$\Lambda$ : conductivity coefficient of moisture

content, $\mathrm{kg} /\left(\mathrm{m} \cdot \mathrm{s} \cdot{ }^{\circ} \mathrm{M}\right)$

\section{Subscripts}

b: bottom surface

$i$ : layer number

$m$ : eigenvalue number

ref: reference value

t: top surface

0: initial

$\infty$ : surrounding medium 


\section{Introduction}

It has long been known that there exists an interdependence between heat and moisture diffusion processes in solids. This interdependence significantly affects the transient distributions of temperature and moisture concentration in some porous materials and resin composites [1]. Moreover, the absorption of moisture by hygroscopic materials in high-temperature environments leads to a considerable increase in hygrothermal stress, which, in turn, degrades the mechanical stiffness and strength of the materials to a large extent [2]. Therefore, it is important to accurately predict the interdependent (coupled) heat and moisture diffusion behaviours in materials and the resulting stress responses in order to assess the soundness of moisture-conditioning building materials and resin-based structural materials in hygrothermal environments. To this end, several researchers have devoted their work to the theoretical understanding of the hygrothermal stress behaviour in hygroscopic materials.

Sih et al. presented analytical or numerical solutions for the coupled heat and moisture diffusion problems [3] as well as the transient hygrothermoelastic problems in the case of plates [4; 5; 6], a strip [7], an infinite body with a spherical cavity [8], and plane bodies containing a circular cavity [6; 9]; they took into account the two-way coupling between heat and moisture. Chang et al. used a decoupling technique to obtain analytical solutions for hygrothermal stresses occurring in a hollow cylinder [1] and a solid cylinder [10] subjected to hygrothermal loadings. Subsequently, using the same technique, Sugano et al. [11; 12] obtained analytical solutions for hygrothermal stresses in a hollow cylinder subjected to non-axisymmetric hygrothermal loading; Chu et al. [13], for hygrothermal stresses in a slab, sphere, and cylinder, under a time-varying boundary condition; and Kishidaira et al. [14], for hygrothermal stresses in an infinite body with a cylindrical hole. In addition, Chen et al. $[15 ; 16]$ analysed the plane hygrothermoelastic problems using the finite element method (FEM). All the papers mentioned above, however, have targeted a single material body.

In contrast, studies related to the coupled hygrothermoelastic problems of layered bodies are limited, despite their industrial importance. Chen et al. [15] analysed the coupled hygrothermal stresses in a double-layered cylinder using the 
FEM; this computation was a time-consuming one. In order to overcome this limitation, Chang et al. [17] later proposed an analytical technique based on the Hankel transform, Laplace transform and its numerical inversion; the computational time was significantly reduced by this technique as compared to that in the case of the FEM analysis. On realizing that hygrothermal stresses became considerably large at the cylinder surfaces immediately after the heat and moisture began to diffuse, they also examined intensively the behaviour of the hygrothermal stresses for a small time interval [18]. However, the continuity conditions at the layer interface and boundary conditions that they used did not include the coupling effects between heat and moisture. Recently, Yang et al. [19] conducted a transient hygrothermoelastic analysis for a single-layered cylinder considering the coupling effects between heat and moisture at the boundaries as well as those within the body. Chiba [20] derived an analytical solution for oneway coupled transient heat and moisture diffusion in a double-layered plate considering the coupling effects included in the boundary conditions and the continuity condition at the layer interface with respect to moisture diffusion. This analytical solution fulfills the condition of exact continuity in moisture flux at the layer interface, a condition that was ignored in existing analytical studies because of mathematical difficulties.

To the best of the authors' knowledge, there appears to be no analytical studies that have dealt with the transient hygrothermal stresses in layered bodies composed of more than three layers. Likewise, the behaviour of the transient hygrothermal stresses in non-homogeneous bodies is yet to be reported, even from the viewpoint of numerical analysis. In the present study, the one-dimensional transient coupled heat and moisture diffusion and the resulting hygrothermal stress field are analysed in a layered plate subjected to hygrothermal loadings at the external surfaces. Analytical solutions for temperature and moisture in multilayered plates are obtained by extending previously derived solutions for doublelayered plates [20]. The diffusion problem considered here is a one-way coupled problem $[21 ; 22 ; 23]$ in which the moisture-induced effects on heat diffusion (i.e. latent heat diffusion) are neglected, but the exact continuity in moisture flux at the layer interfaces is taken into account. An analytical solution for hygrothermal stresses is also derived by the simple superposition of solutions for thermal stresses and hygrostresses. 
First, numerical calculations are performed for a double-layered plate composed of distinct resin-based composites to investigate the influence of the continuity in moisture flux at the layer interface on the hygrothermal stress distribution. Second, the solutions obtained are applied to the hygrothermoelastic problem of a functionally graded material-like (FGM-like) non-homogeneous plate (whose physical properties vary arbitrarily along the thickness direction) composed of hygroscopic materials. Numerical results reveal the hygrothermal stress relaxation induced by a gradual change in the material composition of the plate.

\section{Theoretical analysis}

\subsection{Temperature and moisture fields}

Consider an infinite multi-layered plate made of hygroscopic materials; in this case, the Cartesian coordinate system is considered, as shown in Fig. 1. The total thickness of the plate composed of $n$ layers is represented by $l$. The subscript $i(=1,2, \ldots, n)$ denotes the $i$ th layer of the multi-layered plate. Note that the coordinate value $a_{i}$ indicates the location of the bottom surface of the $i$ th layer.

For the sake of convenience in solving the moisture diffusion equation for a layered body, the concept of moisture potential is used. The temperature and moisture potential of the plate are assumed to be initially uniform throughout the plate thickness and given by $T_{0}$ and $u_{0}$, respectively. The temperatures of the surrounding media are denoted by functions $T_{\mathrm{t} \infty}(t)$ and $T_{\mathrm{b} \infty}(t)$, and the moisture potentials of them by $u_{\mathrm{t} \infty}(t)$ and $u_{\mathrm{b} \infty}(t)$. The plate is subjected to hygrothermal loadings via heat and moisture transfer coefficients $h_{\mathrm{t}}, h_{\mathrm{b}}$ and $\chi_{\mathrm{t}}, \chi_{\mathrm{b}}$, respectively.

For the one-dimensional case shown in Fig. 1, heat and moisture move only along the $z$-axis. When the effect of moisture potential gradient in the energy equation is neglected, the transient heat and moisture diffusion equations for the $i$ th layer are expressed in a dimensionless form, as follows [24]:

$$
\frac{1}{\bar{\kappa}_{i}} \frac{\partial \bar{T}_{i}(Z, \tau)}{\partial \tau}=\frac{\partial^{2} \bar{T}_{i}(Z, \tau)}{\partial Z^{2}} \quad \tau>0
$$




$$
\frac{1}{L_{i}} \frac{\partial \bar{u}_{i}(Z, \tau)}{\partial \tau}=\frac{\partial^{2} \bar{u}_{i}(Z, \tau)}{\partial Z^{2}}-P_{i} \frac{\partial^{2} \bar{T}_{i}(Z, \tau)}{\partial Z^{2}} \quad \tau>0
$$

For constant moisture capacity, the moisture potential $u_{i}$ and moisture content $m_{i}$ are related by $m_{i}=c_{i} \cdot u_{i}$. The one-way coupled system of equations given by Eqs. (1a) and (1b) is equivalent to the constant properties model presented by Fudym et al. [21]. In several practical cases, latent heat term, which is given by the second term on the right-hand side of Lykov's original energy equation [24], has little effect on transient heat and moisture transfer $[12 ; 22 ; 23 ; 25]$. Hence, the simplification of a fully coupled system into a one-way coupled system by dropping this term yields acceptable analysis results for not only the temperature and moisture distributions but also the resulting hygrothemal stress distribution.

The initial conditions are defined as

$$
\bar{T}_{i}(Z, 0)=0 ; \bar{u}_{i}(Z, 0)=0 . \quad(2 \mathrm{a}, \mathrm{b})
$$

On two sides of the multi-layered plate $(Z=0$ and $Z=1)$, the mass diffusion due to temperature and moisture gradients affects the mass balance [26]. At each of the interface between two adjacent layers of the plate, the distributions of temperature and moisture potential are continuous, and the moisture flux, which depends on both the temperature and moisture potential gradients, and heat flux must also be continuous. Therefore, the boundary and continuity conditions can be expressed as follows:

$$
\begin{gathered}
\frac{\partial \bar{T}_{1}(0, \tau)}{\partial Z}+B_{\mathrm{t}}\left[\bar{T}_{\mathrm{t} \infty}(\tau)-\bar{T}_{1}(0, \tau)\right]=0 \\
-\frac{\partial \bar{u}_{1}(0, \tau)}{\partial Z}+P_{1} \frac{\partial \bar{T}_{1}(0, \tau)}{\partial Z}-B_{\mathrm{t}}^{*}\left[\bar{u}_{\mathrm{t} \infty}(\tau)-\bar{u}_{1}(0, \tau)\right]=0 \\
\frac{\partial \bar{T}_{n}(1, \tau)}{\partial Z}-B_{\mathrm{b}}\left[\bar{T}_{\mathrm{b} \infty}(\tau)-\bar{T}_{n}(1, \tau)\right]=0 \\
-\frac{\partial \bar{u}_{n}(1, \tau)}{\partial Z}+P_{n} \frac{\partial \bar{T}_{n}(1, \tau)}{\partial Z}+B_{\mathrm{b}}^{*}\left[\bar{u}_{\mathrm{b} \infty}(\tau)-\bar{u}_{n}(1, \tau)\right]=0 \\
\bar{T}_{i}\left(Z_{i}, \tau\right)=\bar{T}_{i+1}\left(Z_{i}, \tau\right) ; \bar{u}_{i}\left(Z_{i}, \tau\right)=\bar{u}_{i+1}\left(Z_{i}, \tau\right) \quad i=1,2, \ldots, n-1,
\end{gathered}
$$




$$
\begin{gathered}
\bar{\lambda}_{i} \frac{\partial \bar{T}_{i}\left(Z_{i}, \tau\right)}{\partial Z}=\bar{\lambda}_{i+1} \frac{\partial \bar{T}_{i+1}\left(Z_{i}, \tau\right)}{\partial Z} \quad i=1,2, \ldots, n-1, \quad(4 \mathrm{c}) \\
-\bar{\Lambda}_{i} \frac{\partial \bar{u}_{i}\left(Z_{i}, \tau\right)}{\partial Z}+\bar{\Lambda}_{i} P_{i} \frac{\partial \bar{T}_{i}\left(Z_{i}, \tau\right)}{\partial Z}=-\bar{\Lambda}_{i+1} \frac{\partial \bar{u}_{i+1}\left(Z_{i}, \tau\right)}{\partial Z}+\bar{\Lambda}_{i+1} P_{i+1} \frac{\partial \bar{T}_{i+1}\left(Z_{i}, \tau\right)}{\partial Z} \\
i=1,2, \ldots, n-1
\end{gathered}
$$

where $Z_{i}=a_{i} / l, \bar{\lambda}_{i}=\lambda_{i} / \lambda_{\text {ref }}$, and $\bar{\Lambda}_{i}=\Lambda_{i} / \Lambda_{\text {ref }}$.

An analytical solution to the transient heat conduction problem given by Eqs. (1a), (2a), (3a), (3c), (4a), and (4c) has already been derived by Sugano et al. [27] as follows:

$$
\bar{T}_{i}(Z, \tau)=\sum_{m=1}^{\infty} \phi_{m}(\tau)\left[A_{i m} \cos \left(\frac{\gamma_{m} Z}{\sqrt{\bar{\kappa}_{i}}}\right)+B_{i m} \sin \left(\frac{\gamma_{m} Z}{\sqrt{\bar{\kappa}_{i}}}\right)\right]+\sum_{j=1}^{2}\left(C_{i j} Z+D_{i j}\right) V_{j}(\tau)
$$

where

$$
\begin{array}{r}
V_{1}(\tau)=-\bar{T}_{\mathrm{t} \infty}(\tau) ; V_{2}(\tau)=\bar{T}_{\mathrm{b} \infty}(\tau), \quad(6 \mathrm{a}, \mathrm{b}) \\
\phi_{m}(\tau)=\exp \left(-\gamma_{m}^{2} \tau\right)\left[g_{m}-\int_{0}^{\tau} \exp \left(\gamma_{m}^{2} t\right) \sum_{j=1}^{2} f_{m j} \frac{\mathrm{d} V_{j}(t)}{\mathrm{d} t} \mathrm{~d} t\right] .
\end{array}
$$

The procedure for determining the eigenvalues $\gamma_{m}(m=1,2, \ldots)$ and the expansion coefficients $g_{m}$ and $f_{m j}(j=1,2)$ can be found in [27]. The constants $A_{i m}, B_{i m}, C_{i j}$, and $D_{i j}$ in Eq. (5) are determined from the boundary and continuity conditions given by Eqs. (3a), (3c), (4a), and (4c).

Combining Eq. (1b) with Eq. (5) yields

$$
\frac{1}{L_{i}} \frac{\partial \bar{u}_{i}(Z, \tau)}{\partial \tau}=\frac{\partial^{2} \bar{u}_{i}(Z, \tau)}{\partial Z^{2}}+Q_{i}(Z, \tau)
$$

where

$$
Q_{i}(Z, \tau)=\frac{P_{i}}{\bar{\kappa}_{i}} \sum_{m=1}^{\infty} \gamma_{m}^{2} \phi_{m}(\tau)\left[A_{i m} \cos \left(\frac{\gamma_{m} Z}{\sqrt{\bar{\kappa}_{i}}}\right)+B_{i m} \sin \left(\frac{\gamma_{m} Z}{\sqrt{\bar{\kappa}_{i}}}\right)\right]
$$

An analytical solution to the transient moisture diffusion problem for a composite medium represented by Eqs. (2b), (3b), (3d), (4b), (4d), and (8) is derived by 
extending the moisture potential solution obtained previously for double-layered plates [20]. The solution is obtained as follows:

$$
\bar{u}_{i}(Z, \tau)=\sum_{m=1}^{\infty} \psi_{m}(\tau) R_{i m}(Z)+\sum_{j=1}^{n+1} F_{i j}(Z) W_{j}(\tau), \quad i=1,2, \ldots, n .
$$

For details of functions $\psi_{m}, R_{i m}, F_{i j}$, and $W_{j}$, refer to the appendix.

\subsection{Hygrothermal stresses}

Once the applied temperature and moisture fields are determined, it is easy to analyse the resulting hygrothermal stresses in accordance with the principle of superposition [6]. An expression for in-plane thermal stresses in plates that are non-homogeneous across the thickness was obtained by Sugano [28] for the case in which the temperature only varied along the thickness direction, that is, $T=$ $T(z)$ and the boundary surfaces were free of tractions. Obviously, this expression is valid for the thermal stresses in the multi-layered plate under consideration, because the plate can be regarded as a non-homogeneous one in which the material properties vary in a stepwise manner along the thickness direction. Moreover, because of an analogy between thermal stress and hygrostress, this expression is also valid for hygrostresses calculated using the coefficient of moisture expansion and moisture content, instead of the coefficient of thermal expansion and temperature.

By the superposition of the expressions for thermal stresses and hygrostresses, the non-zero stress components in a multi-layered plate whose surfaces are free of tractions are given by

$$
\begin{aligned}
\sigma_{x x}=\sigma_{y y}=\frac{E(Z)}{1-v(Z)}\{- & {[\alpha(Z) \Delta T(Z, \tau)+\beta(Z) \Delta m(Z, \tau)] } \\
& \left.+\frac{\left(I_{2} Z-I_{3}\right) \Phi_{1}(\tau)+\left(I_{2}-I_{1} Z\right) \Phi_{2}(\tau)}{I_{2}^{2}-I_{1} I_{3}}\right\},
\end{aligned}
$$

where

$$
I_{j}=\int_{0}^{1} \frac{Z^{j-1} E(Z)}{1-v(Z)} \mathrm{d} Z, \quad j=1,2,3
$$




$$
\Phi_{j}(\tau)=\int_{0}^{1} \frac{Z^{j-1} E(Z)[\alpha(Z) \Delta T(Z, \tau)+\beta(Z) \Delta m(Z, \tau)]}{1-v(Z)} \mathrm{d} Z, \quad j=1,2
$$

with $\Delta T=T-T_{0}$ and $\Delta m=m-m_{0}$.

\section{Numerical results and discussion}

\subsection{Case study 1: double-layered plate}

Numerical calculations are performed for a double-layered plate composed of a T300/5208 composite (layer 1) and another hygroscopic material (layer 2). The thickness of both the layers is assumed to be identical, that is, $Z_{1}=0.5$. The material parameters used in the heat and moisture diffusion analysis and the stress analysis are as follows $[15 ; 18 ; 19 ; 29]$ :

$$
\begin{aligned}
& \alpha_{1}=\alpha_{2}=31.3 \times 10^{-6} \mathrm{~cm} /(\mathrm{cm} \cdot \mathrm{K}), \quad \beta_{1}=\beta_{2}=2.68 \times 10^{-3} \mathrm{~cm} /\left(\mathrm{cm} \cdot \% \mathrm{H}_{2} \mathrm{O}\right), \\
& v_{1}=v_{2}=0.33, \quad \kappa_{1}=5 \kappa_{2}=2.16 \times 10^{-5} \mathrm{~m}^{2} / \mathrm{s}, \quad \eta_{1}=\eta_{2}=2.16 \times 10^{-6} \mathrm{~m}^{2} / \mathrm{s}, \\
& E_{1}=64.3 \mathrm{GPa}, \quad E_{2}=79.7 \mathrm{GPa}, \quad c_{1}=5 c_{2}=0.01 \mathrm{~kg} /\left(\mathrm{kg} \cdot{ }^{\circ} \mathrm{M}\right), \\
& \lambda_{1}=5 \lambda_{2}=0.65 \mathrm{~W} /(\mathrm{m} \cdot \mathrm{K}), \quad \varepsilon_{1}=\varepsilon_{2}=2.0^{\circ} \mathrm{M} / \mathrm{K} \\
& \Lambda_{1}=\Lambda_{2}=2.2 \times 10^{-8} \mathrm{~kg} /\left(\mathrm{m} \cdot \mathrm{s} \cdot{ }^{\circ} \mathrm{M}\right) .
\end{aligned}
$$

The material properties of the layer 1 are used as a reference for the dimensionless quantities. We consider the case in which the same temperature and moisture potential values are prescribed at both the external surfaces of the double-layered plate, i.e. $B_{\mathrm{t}}=B_{\mathrm{b}}=B_{\mathrm{t}}^{*}=B_{\mathrm{b}}^{*}=\infty, \bar{T}_{\mathrm{t} \infty}=\bar{T}_{\mathrm{b} \infty}=1$, and $\bar{u}_{\mathrm{t} \infty}=\bar{u}_{\mathrm{b} \infty}=1$ for $\left(u_{0}-u_{\text {ref }}\right) /\left(T_{\text {ref }}-T_{0}\right)=0.82^{\circ} \mathrm{M} / \mathrm{K}[30]$; this case represents symmetric drying with heating. The number of terms in the infinite series in Eqs. (5) and (10) is taken as 100 , which is used to ensure sufficient convergence of the numerical results.

The transient temperature and moisture potential distributions in this case are given in [20]. In this reference, the continuity in moisture flux at the layer interface, which was inappropriately formulated in existing analytical studies, is correctly formulated, and its effect on transient moisture potential distribution is shown to be pronounced. In contrast, in the present study, the influence of 
moisture-flux continuity upon transient hygrothermal stress distribution is investigated.

The transient hygrothermal stress distributions shown in Fig. 2 are divided into two groups: one of the groups is composed of those computed on the basis of the exact continuity condition, given by Eq. (4d); the other is composed of those based on the simplified continuity condition such that the second term on both the sides of Eq. (4d) is omitted, which was commonly used in existing analytical studies. In Fig. 2, some differences that cannot be neglected quantitatively have been found between both the groups for all elapsed times except for very short or very long ones, although differences between the distribution tendencies of both the groups are not notable. Furthermore, for any given elapsed time, the maximum tensile stress that may trigger material failure is lower in the case represented by Eq. (4d). In both the groups, a large stress discontinuity is induced at the layer interface despite the fact that both the layers have identical coefficients of moisture expansion and thermal expansion. This is because under hygrothermal equilibrium, the moisture potential of two bodies in contact is the same but the moisture content of both undergoes a rapid change at the boundary of contact.

\subsection{Case study 2: FGM-like non-homogeneous plate}

In the second case study, numerical calculations are performed for FGM-like non-homogeneous plates composed of the two materials mentioned above. The volume fraction distribution of one of the constituents (T300/5208 composite), $V_{\mathrm{C}}(Z)$, is shown in Fig. 3. As shown in the figure, numerical calculations are conducted for three types of graded material composition for which $V_{\mathrm{C}}(Z)$ is expressed by the power functions of $Z$ as

$$
V_{\mathrm{C}}(Z)=(1-Z)^{N}
$$

In the numerical calculations, we approximately express the continuous spatial changes in the volume fraction of the non-homogenous plates by means of the layered-plate model consisting of 50 layers with different volume fractions, i.e., $n$ $=50$. In other words, we consider the volume fraction variations shown in Fig. 3 as stepwise variations with 50 steps. 
The effective material properties of the FGM-like non-homogeneous plates are evaluated according to the linear rule of mixture for simplicity, although one can also evaluate them using various micromechanics-based formulations such as the Mori-Tanaka method and the self-consistent method. Thus, each effective material property $P^{\prime}$ is given as

$$
P^{\prime}(Z)=P_{\mathrm{C}}^{\prime} V_{\mathrm{C}}(Z)+P_{\mathrm{O}}^{\prime}\left[1-V_{\mathrm{C}}(Z)\right]
$$

where $P_{\mathrm{C}}^{\prime}$ denotes the individual material property of T300/5208 composite, and $P_{\mathrm{O}}^{\prime}$ denotes that of the other constituent material.

In this case study, the material properties of the T300/5208 composite are used for nondimensionalisation. The plates are subjected to hygrothermal loadings such that $\bar{T}_{\mathrm{t} \infty}=\bar{u}_{\mathrm{t} \infty}=0$, while the other parameters remain unchanged from the first case study. Hence, these unsymmetrical boundary conditions give a steadystate temperature distribution having non-zero gradients unlike the first case study.

Figure 4 shows the transient temperature, moisture potential, and resulting hygrothermal stress distributions for a material gradation index $N=1$. The temperature distribution increases monotonously with time. Although the moisture potential distributions also vary in a relatively simple fashion, a characteristic region in which the moisture potential values fall depending on the curves of temperature distributions exists; this is because the second derivative of temperature acts as the moisture sink (see Eq. (1b)). Over time, this region moves towards the top surface of the plate $(Z=0)$.

The effect of the continuity condition for moisture flux is noticeable in this case study as well. The difference between the moisture potential distributions computed on the basis of different continuity conditions increases with time. In the steady state, the effects of the moisture sink term in Eq. (1b) vanish, and the moisture potential distribution is linear under the simplified continuity condition because the values of $\Lambda$ are the same for the two constituents of the nonhomogeneous plates, as shown in Eq. (12). On the other hand, under the exact continuity condition, given by Eq. (4d), the steady-state distribution is a concave upward curve, which is different from the straight line obtained under the simplified continuity condition. Consequently, such a large difference in the moisture potential distribution directly leads to a significant difference in the 
steady-state hygrothermal stress distribution. In fact, under the simplified continuity condition, the steady-state maximum tensile and compressive stresses are both overestimated to a great extent as compared to those under the exact continuity condition.

Moreover, from a comparison between Fig. 2 and Fig. 4 (c), the following trend can be found: in cases in which the temperature gradient converges to zero with elapsed time (e.g., case study 1), the distributions of hygrothermal stresses do not differ so much depending on whether the continuity conditions of moisture flux is simplified or not; on the other hand, in cases in which the temperature gradient develops with time in a wide region to yield a large temperature gradient at the steady state (e.g., case study 2), a pronounced difference due to the right and wrong of the continuity conditions is observed in the steady-state hygrothermal stress distributions. This leads to a finding that the exact continuity conditions of Eq. (4d) are strongly required in the latter hygrothermoelastic problems.

Based on Figs. 5-7, a comparison is made between the results attained for different material gradation indices. The temperature and moisture potential of the non-homogeneous plates and the double-layered plate are calculated on the basis of the continuity condition represented by Eq. (4d), and their contour plots are presented in Figs. 5 and 6, respectively. It can be observed that the temperatures increase along the $Z$ axis in all the plots, although the rates of increase depend on the spatial profiles of the material composition of the plate. In the nonhomogeneous plate with $N=3$, since the volume fraction of T300/5208 composite, which is a constituent material with higher thermal diffusivity, is totally low, the temperature variation is slower than in the plate with $N=1 / 3$. In addition, the double-layered plate has a thick layer of material with low thermal conductivity on the bottom side (heating side) and this is a very similar condition to the non-homogeneous plate with $N=3$, thereby resulting in almost the same temperature distribution between them until $\tau$ reaches about 0.2 . After that, an obvious discontinuity in the temperature gradient is observed at the interface in the double-layered plate. The content of T300/5208 composite in the doublelayered plate is identical to that in the non-homogeneous plate with $N=1$ (linear material composition distribution). However, the thermal barrier performance of the double-layered plate is found to be considerably better than that of the non- 
homogeneous plate with $N=1$ because the transient behaviour of the temperature in the non-homogeneous plate with $N=1$ (see Fig. 4(a)) is intermediate between Fig. 5 (a) and (b).

The transient behaviour of moisture potential is more complicated than that of temperature. Except in the steady state of the non-homogeneous plate with $N=$ $1 / 3$, the moisture potentials decrease along the $Z$ axis to exhibit minimal values, and then increase along the $Z$ axis at a gradually increasing rate. In the contour plot for the double-layered plate, there is a wide region where the moisture potential is negative, and the absolute values of these negative values are higher than those in the contour plots for the non-homogeneous plates. Furthermore, the moisture potential distributions differ substantially between the non-homogeneous plates with different material composition and between the non-homogeneous plate and double-layered plate. Since the values of $\eta, \Lambda$ and $\varepsilon$ are identical among the constituent materials of the three plates, the reason of this is purely because the second term on right hand side of Eq. (1b) and the second terms on both sides of Eq. (4d), i.e., temperature gradients, affect the moisture potential distributions. According to Eq. (1b), more convex downward temperature distribution leads to larger moisture sink, and therefore the non-homogeneous plate with $N=3$, which has a more convex downward temperature distribution, produces more decrease in the moisture potential than the non-homogeneous plate with $N=1 / 3$.

The moisture potentials are partly negative at the steady state, at which the moisture sink term in the governing equation vanishes, just because of the temperature gradients in Eq. (4d). In the non-homogeneous plate with $N=1 / 3$, however, the steady-state moisture potential is not negative anywhere. This is because the steady-state temperature distribution is approximately-linear (see Fig. 5 (b)) and the effects of temperature gradient terms in Eq. (4d) become negligible by compensating them between right and left sides of the equation in the case where $\Lambda$ and $\varepsilon$ (or $P$ ) are constant throughout the plate-thickness, as the present numerical example.

In the 3D graphs of the hygrothermal stresses shown in Fig. 7, the maximum compressive stresses occur at the bottom surface of the plate $(Z=1)$ immediately after the heat and moisture begin to diffuse, whereas the maximum tensile stresses tend to occur within the plate in the unsteady state, and not in the steady state. It is interesting that the maximum tensile or compressive stresses are 
observed at the same through-thickness location in all the three plates. Although not shown in the figure, for $N=1, \bar{\sigma}_{\max }=0.2789$ at $(Z, \tau)=(0.66,0.05)$ and $\bar{\sigma}_{\text {min }}=-1.4555$ at $(Z, \tau)=(1,0.001)$. However, the fact that the maximum tensile stresses occur at almost the same location independently of the material composition distribution may be just a coincidence, and it is probably peculiar to the present numerical example. A characteristic rise and fall in the hygrothermal stress is observed at $\tau \cong 1$ in the neighborhood of the plate surfaces; this would be due to a peculiar change in the hygrostress caused by the vanishment of the moisture sink term in Eq. (1b) at the moment when the temperature field reaches the steady state. Moreover, as compared to the double-layered plate, the FGM-like non-homogeneous plates, especially the one with $N=1 / 3$, exhibit small maximum tensile and compressive stresses, and considerable hygrothermal stress relaxation (e.g., approximately $21 \%$ of maximum tensile stress relaxation for $N=1 / 3$ ) is induced by a gradual change in the material composition.

\section{Conclusions}

In the present study, analytical solutions have been presented for the transient one-way coupled temperature and moisture fields and the resulting hygrothermal stresses in a multi-layered plate subjected to hygrothermal loadings at the external surfaces. Subsequently, the effects of the coupling terms included in the continuity conditions for moisture fluxes at the layer interfaces on the hygrothermal stress distribution in the plate have been investigated. Moreover, the solutions presented have been applied to the hygrothermoelastic problem of FGMlike non-homogeneous plates made of hygroscopic materials; the physical properties of these plates vary arbitrarily along the thickness direction.

Numerical results have shown that, in order to avoid significant errors in evaluating the transient hygrothermal stress behaviour of a layered body, it is necessary to consider the moisture flux component that results from the temperature gradient in the continuity conditions of moisture flux at the layer interfaces. Additionally, a comparison of stress distributions between the FGMlike non-homogeneous plates and double-layered plate has demonstrated the hygrothermal stress relaxation induced by a gradual change in the material composition of the non-homogeneous plates. 


\section{Acknowledgement}

This work was financially supported by KAKENHI (14919018: Grant-in-Aid for Encouragement of Scientists). 


\section{Appendix}

Details of the functions in Eq. (10) are as follows:

$$
\begin{gathered}
F_{i j}(Z)=C_{i j}^{*} Z+D_{i j}^{*}, \quad j=1,2, \ldots, n+1, \\
W_{1}(\tau)=\frac{P_{1}}{B_{\mathrm{t}}^{*}} \frac{\partial \bar{T}_{1}(0, \tau)}{\partial Z}-\bar{u}_{\mathrm{t} \infty}(\tau) \text { for } B_{\mathrm{t}}^{*} \neq 0, \quad W_{1}(\tau)=0 \text { for } B_{\mathrm{t}}^{*}=0, \quad \text { (A.2a) } \\
\left.W_{n+1}(\tau)=\frac{P_{n}}{B_{\mathrm{b}}^{*}} \frac{\partial \bar{T}_{n}(1, \tau)}{\partial Z}+\bar{u}_{\mathrm{b} \infty}(\tau) \text { for } B_{\mathrm{b}}^{*} \neq 0, \quad W_{n+1}(\tau)=0 \text { for } B_{\mathrm{b}}^{*}=0, \quad \text { (A. } 2 \mathrm{~b}\right) \\
\left.W_{j}(\tau)=\bar{\Lambda}_{j-1} P_{j-1} \frac{\partial \bar{T}_{j-1}\left(Z_{j-1}, \tau\right)}{\partial Z}-\bar{\Lambda}_{j} P_{j} \frac{\partial \bar{T}_{j}\left(Z_{j-1}, \tau\right)}{\partial Z}, j=2,3, \ldots, n-1, n . \quad \text { (A. } 2 \mathrm{c}\right)
\end{gathered}
$$

Constants $C_{i j}^{*}$ and $D_{i j}^{*}$ are determined according to the following relationships:

$$
\begin{gathered}
F_{i j}\left(Z_{i}\right)-F_{(i+1) j}\left(Z_{i}\right)=0, \quad i=1,2, \ldots, n-1, \quad j=1,2, \ldots, n+1, \quad \text { (A.3a) } \\
\bar{\Lambda}_{i} \frac{\mathrm{d} F_{i j}\left(Z_{i}\right)}{\mathrm{d} Z}-\bar{\Lambda}_{i+1} \frac{\mathrm{d} F_{(i+1) j}\left(Z_{i}\right)}{\mathrm{d} Z}=\delta_{(i+1), j} \quad i=1,2, \ldots, n-1, \quad j=1,2, \ldots, n+1,(\mathrm{~A} \\
\frac{\mathrm{d} F_{1 j}(0)}{\mathrm{d} Z}-B_{\mathrm{t}}^{*} F_{1 j}(0)=B_{\mathrm{t}}^{*} \delta_{1, j}, \quad j=1,2, \ldots, n+1, \quad \text { (A.3c) } \\
\frac{\mathrm{d} F_{n j}(1)}{\mathrm{d} Z}+B_{\mathrm{b}}^{*} F_{n j}(1)=B_{\mathrm{b}}^{*} \delta_{(n+1), j}, \quad j=1,2, \ldots, n+1 . \quad \text { (A.3d) }
\end{gathered}
$$

Function $R_{i m}(Z)$ is the solution to the eigenvalue problem corresponding to Eqs. (3b), (3d), (4b), (4d), and (8) and is given as follows:

$$
R_{i m}(Z)=A_{i m}^{*} \cos \left(\frac{\mu_{m} Z}{\sqrt{L_{i}}}\right)+B_{i m}^{*} \sin \left(\frac{\mu_{m} Z}{\sqrt{L_{i}}}\right)
$$

where $\mu_{m}$ is an eigenvalue. The conditions necessary to determine the unknown constants $A_{i m}^{*}$ and $B_{i m}^{*}$ can be obtained by substituting Eqs. (10) and (A.1)-(A.4) into Eqs. (3b), (3d), (4b), and (4d) as follows:

$$
\frac{\mathrm{d} R_{1 m}(0)}{\mathrm{d} Z}-B_{\mathrm{t}}^{*} R_{1 m}(0)=0
$$




$$
\begin{gathered}
\frac{\mathrm{d} R_{n m}(1)}{\mathrm{d} Z}+B_{\mathrm{b}}^{*} R_{n m}(1)=0, \quad \text { (A.5b) } \\
R_{i m}\left(Z_{i}\right)=R_{(i+1) m}\left(Z_{i}\right), \quad i=1,2, \ldots, n-1, \\
\bar{\Lambda}_{i} \frac{\mathrm{d} R_{i m}\left(Z_{i}\right)}{\mathrm{d} Z}=\bar{\Lambda}_{i+1} \frac{\mathrm{d} R_{(i+1) m}\left(Z_{i}\right)}{\mathrm{d} Z} \quad i=1,2, \ldots, n-1 .
\end{gathered}
$$

Eigenvalues $\mu_{m}(m=1,2, \ldots)$ are obtained from the condition under which all the $A_{i m}^{*}$ and $B_{i m}^{*}$ values are non-zero and are, therefore, positive roots of the following transcendental equation:

$$
\mathbf{G} \cdot \mathbf{E}_{1} \cdot \mathbf{E}_{2} \cdots \mathbf{E}_{n-1} \cdot \mathbf{a}=0, \quad \text { (A.6) }
$$

where

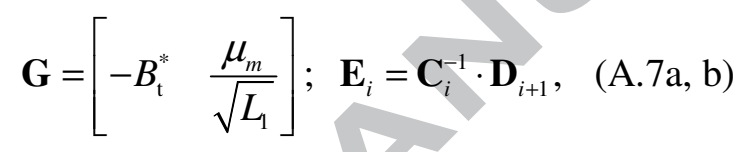

$$
\begin{aligned}
& \mathbf{C}_{i}=\left[\begin{array}{cc}
\cos \left(\frac{\mu_{m} Z_{i}}{\sqrt{L_{i}}}\right) & \sin \left(\frac{\mu_{m} Z_{i}}{\sqrt{L_{i}}}\right) \\
-\bar{\Lambda}_{i} \frac{\mu_{m}}{\sqrt{L_{i}}} \sin \left(\frac{\mu_{m} Z_{i}}{\sqrt{L_{i}}}\right) & \bar{\Lambda}_{i} \frac{\mu_{m}}{\sqrt{L_{i}}} \cos \left(\frac{\mu_{m} Z_{i}}{\sqrt{L_{i}}}\right)
\end{array}\right], \\
& \mathbf{D}_{i+1}=\left[\begin{array}{cc}
\cos \left(\frac{\mu_{m} Z_{i}}{\sqrt{L_{i+1}}}\right) & \sin \left(\frac{\mu_{m} Z_{i}}{\sqrt{L_{i+1}}}\right) \\
-\bar{\Lambda}_{i+1} \frac{\mu_{m}}{\sqrt{L_{i+1}}} \sin \left(\frac{\mu_{m} Z_{i}}{\sqrt{L_{i+1}}}\right) & \bar{\Lambda}_{i+1} \frac{\mu_{m}}{\sqrt{L_{i+1}}} \cos \left(\frac{\mu_{m} Z_{i}}{\sqrt{L_{i+1}}}\right)
\end{array}\right] \\
& \mathbf{a}=\left[\begin{array}{l}
\frac{\mu_{m}}{\sqrt{L_{n}}} \cos \left(\frac{\mu_{m}}{\sqrt{L_{n}}}\right)+B_{\mathrm{b}}^{*} \sin \left(\frac{\mu_{m}}{\sqrt{L_{n}}}\right) \\
\frac{\mu_{m}}{\sqrt{L_{n}}} \sin \left(\frac{\mu_{m}}{\sqrt{L_{n}}}\right)-B_{\mathrm{b}}^{*} \cos \left(\frac{\mu_{m}}{\sqrt{L_{n}}}\right)
\end{array}\right] .
\end{aligned}
$$

Function $\psi_{m}(\tau)$ is expressed as

$$
\psi_{m}(\tau)=\exp \left(-\mu_{m}^{2} \tau\right)\left\{g_{m}^{*}+\int_{0}^{\tau} \exp \left(\mu_{m}^{2} t\right)\left[q_{m}(t)-\sum_{j=1}^{n+1} f_{m j}^{*} \frac{\mathrm{d} W_{j}(t)}{\mathrm{d} t}\right] \mathrm{d} t\right\}
$$


where the expansion coefficients $g_{m}^{*}, q_{m}(\tau)$, and $f_{m j}^{*}$ are given by

$$
\begin{gathered}
g_{m}^{*}=-\frac{\sum_{i=1}^{n} \frac{\bar{\Lambda}_{i}}{L_{i}} \int_{Z_{i-1}}^{Z_{i}}\left[\sum_{j=1}^{n+1} F_{i j}(z) W_{j}(0)\right] R_{i m}(z) \mathrm{d} z}{\sum_{i=1}^{n} \frac{\bar{\Lambda}_{i}}{L_{i}} \int_{Z_{i-1}}^{Z_{i}}\left[R_{i m}(z)\right]^{2} \mathrm{~d} z}, \\
q_{m}(\tau)=\frac{\sum_{i=1}^{n} \bar{\Lambda}_{i} \int_{Z_{i-1}}^{Z_{i}} Q_{i}(z, \tau) R_{i m}(z) \mathrm{d} z}{\sum_{i=1}^{n} \frac{\bar{\Lambda}_{i}}{L_{i}} \int_{Z_{i-1}}^{Z_{i}}\left[R_{i m}(z)\right]^{2} \mathrm{~d} z}, \\
f_{m j}^{*}=\frac{\sum_{i=1}^{n} \frac{\bar{\Lambda}_{i}}{L_{i}} \int_{Z_{i-1}}^{Z_{i}} F_{i j}(z) R_{i m}(z) \mathrm{d} z}{\sum_{i=1}^{n} \frac{\bar{\Lambda}_{i}}{L_{i}} \int_{Z_{i-1}}^{Z_{i}}\left[R_{i m}(z)\right]^{2} \mathrm{~d} z} .
\end{gathered}
$$

Function $Q_{i}(z, \tau)$ in Eq. (A.9b) is given by Eq. (9). 


\section{References}

[1] Chang WJ, Chen TC, Weng CI. Transient hygrothermal stresses in an infinitely long annular cylinder: coupling of heat and moisture. J Thermal Stress 1991;14(4):439-454.

[2] Komai K, Minoshima K, Shiroshita S. Hygrothermal degradation and fracture process of advanced fibre-reinforced plastics. Mater. Sci. Eng. A 1991;143(1-2):155-166.

[3] Hartranft RJ, Sih GC. The influence of the Soret and Dufour effects on the diffusion of heat and moisture in solids. Int. J. Eng. Sci. 1980 18(12):1375-1383.

[4] Sih GC, Shih MT, Chou SC. Transient hygrothermal stresses in composites: coupling of moisture and heat with temperature varying diffusivity. Int. J. Eng. Sci. 1980;18(1):19-42.

[5] Hartranft RJ, Sih GC. The influence of coupled diffusion of heat and moisture on the state of stress in a plate. Mech. Composite Mater. 1980;16(1):44-56.

[6] Sih GC. Transient hygrothermal stresses in plates with and without cavities. Fibre Sci. Tech. 1983;18(3):181-201.

[7] Sih GC, Ogawa A. Transient thermal change on a solid surface: coupled diffusion of heat and moisture. J. Thermal Stress 1982;5(3):265-282.

[8] Hartranft RJ, Sih GC. Stresses induced in an infinite medium by the coupled diffusion of heat and moisture from a spherical hole. Eng. Fracture Mech. 1981;14(2):261-287.

[9] Sih GC, Ogawa A, Chou SC. Two-dimensional transient hygrothermal stresses in bodies with circular cavities: moisture and temperature coupling effects. J. Thermal Stress 1981;4(2):193222.

[10] Chang WJ. Transient hygrothermal responses in a solid cylinder by linear theory of coupled heat and moisture. Appl. Math. Model. 1994;18(8):467-473.

[11] Sugano Y, Chuuman Y. An analytical solution of hygrothemoelastic problem in multiply connected region. Trans. Jpn Soc. Mech. Eng. A 1993;59(562):1519-1525. in Japanese

[12] Sugano Y, Chuuman Y. Analytical solution of transient hygrothermoelastic problem due to coupled heat and moisture diffusion in a hollow circular cylinder. Trans. Jpn Soc. Mech. Eng. A 1993;59(564):1956-1963. in Japanese

[13] Chu JL, Lee S. Hygrothermal stresses in a solid: constant surface stress. J. Appl. Phys. 1993;74(1):171-188.

[14] Kishidaira K, Watanabe K. Hygrothermal stress of clay in a high-speed drying furnace. J. Thermal Stress 1996;19(7):599-612.

[15] Chen TC, Weng CI, Chang WJ. Transient hygrothermal stresses induced in general plane problems by theory of coupled heat and moisture. Trans. ASME J Appl Mech 1992;59(2S):S10-S16.

[16] Chen TC, Hwang BH. Transient hygrothermal stresses induced in two-dimensional problems by nonlinear theory of coupled heat and moisture. Trans. ASME J. Appl. Mech.

1994;61(4):938-943. 
[17] Chang WJ, Weng CI. An analytical solution of a transient hygrothermal problem in an axisymmetric double-layer annular cylinder by linear theory of coupled heat and moisture. Appl. Math. Model. 1997;21(11):721-734.

[18] Chang WJ, Weng CI. Small time behavior of hygrothermal stresses in axisymmetric doublelayer cylinders. J. Thermal Stress 2000;23(1):15-46.

[19] Yang YC, Chu SS, Lee HL, Lin SL. Hybrid numerical method applied to transient hygrothermal analysis in an annular cylinder. Int. Comm. Heat Mass Transfer 2006;33(1);102111.

[20] Chiba R. An analytical solution for transient heat and moisture diffusion in a double-layer plate. In: Aziz Belmiloudi, editor. Open access edited book: Heat transfer - Mathematical modelling, numerical methods and information technology. Vienna: INTECH, 2011: 567-578. Available from: http://www.intechopen.com/download/pdf/pdfs_id/13431

[21] Fudym O, Batsale JC, Santander R, Bubnovich V. Analytical solution of coupled diffusion equations in semi-infinite media. Trans. ASME J. Heat Transfer 2004;126(3):471-475.

[22] Khoshbakht M, Lin MW, Feickert CA. A finite element model for hygrothermal analysis of masonry walls with FRP reinforcement. Finite Elem. Anal. Des. 2009;45(8-9):511-518.

[23] Khoshbakht M, Lin MW. A finite element model for hygro-thermo-mechanical analysis of masonry walls with FRP reinforcement. Finite Elem. Anal. Des. 2010;46(10):783-791.

[24] Lykov AV, Mikhailov YA. Theory of heat and mass transfer. Jerusalem: Israel Program for Scientific Translations, 1965.

[25] Kulasiri D, Woodhead I. On modelling the drying of porous materials: analytical solutions to coupled partial differential equations governing heat and moisture transfer. Math. Probl. Eng. 2005;2005(3):275-291.

[26] Chang WJ, Weng CI. An analytical solution to coupled heat and moisture diffusion transfer in porous materials. Int. J. Heat Mass Transfer 2000;43(19):3621-3632.

[27] Sugano Y, Morishita H, Tanaka K. An analytical solution for transient thermal stress in a functionally gradient plate with arbitrary nonhomogeneities and thermal boundary conditions: material properties determined by fuzzy inference. Trans. Jpn Soc. Mech. Eng. A 1993;59(567):2666-2673. in Japanese

[28] Sugano Y. An expression for transient thermal stress in a nonhomogeneous plate with temperature variation through thickness. Arch. Appl. Mech. 1987;57(2):147-156.

[29] Sih GC, Michopoulos JG, Chou SC. Hygrothermoelasticity. Dordrecht: Martinus Nijhoff Publishers, 1986.

[30] Liu JY, Shun C. Solutions of Luikov equations of heat and mass transfer in capillary-porous bodies. Int. J. Heat Mass Transfer 1991;34(7):1747-1754. 


\section{Figure captions}

Fig. 1 Physical model and coordinate system

Fig. 2 Transient behaviour of hygrothermal stresses in a double-layered plate

Fig. 3 Volume fraction profiles of T300/5208 composite in FGM-like non-homogeneous plates

Fig. 4 Transient behaviour of (a) temperature, (b) moisture potential and (c) hygrothermal stresses in a non-homogeneous plate with $N=1$

Fig. 5 Contour plots of transient temperature in non-homogeneous plates with (a) $N=3$ and (b) $N$ $=1 / 3$ and $(\mathrm{c})$ in a double-layered plate

Fig. 6 Contour plots of transient moisture potential in non-homogeneous plates with (a) $N=3$ and (b) $N=1 / 3$ and (c) in a double-layered plate

Fig. 7 Transient hygrothermal stress distributions in non-homogeneous plates with (a) $N=3$ and (b) $N=1 / 3$ and (c) in a double-layered plate 


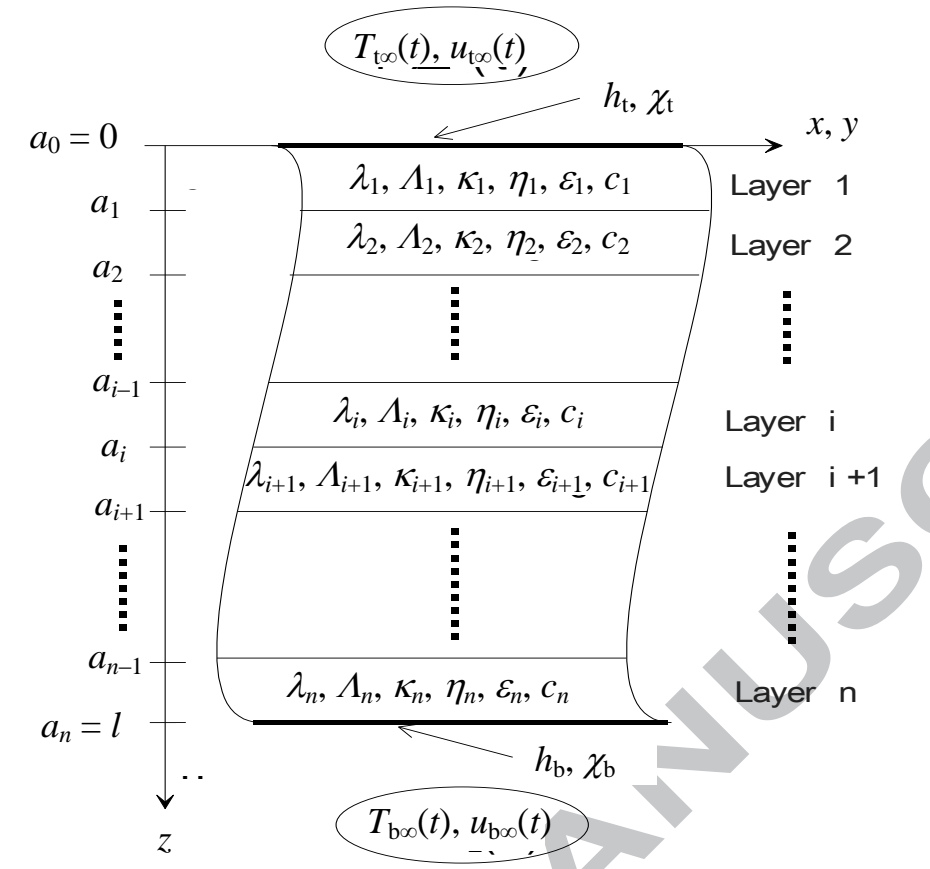

Fig. 1 


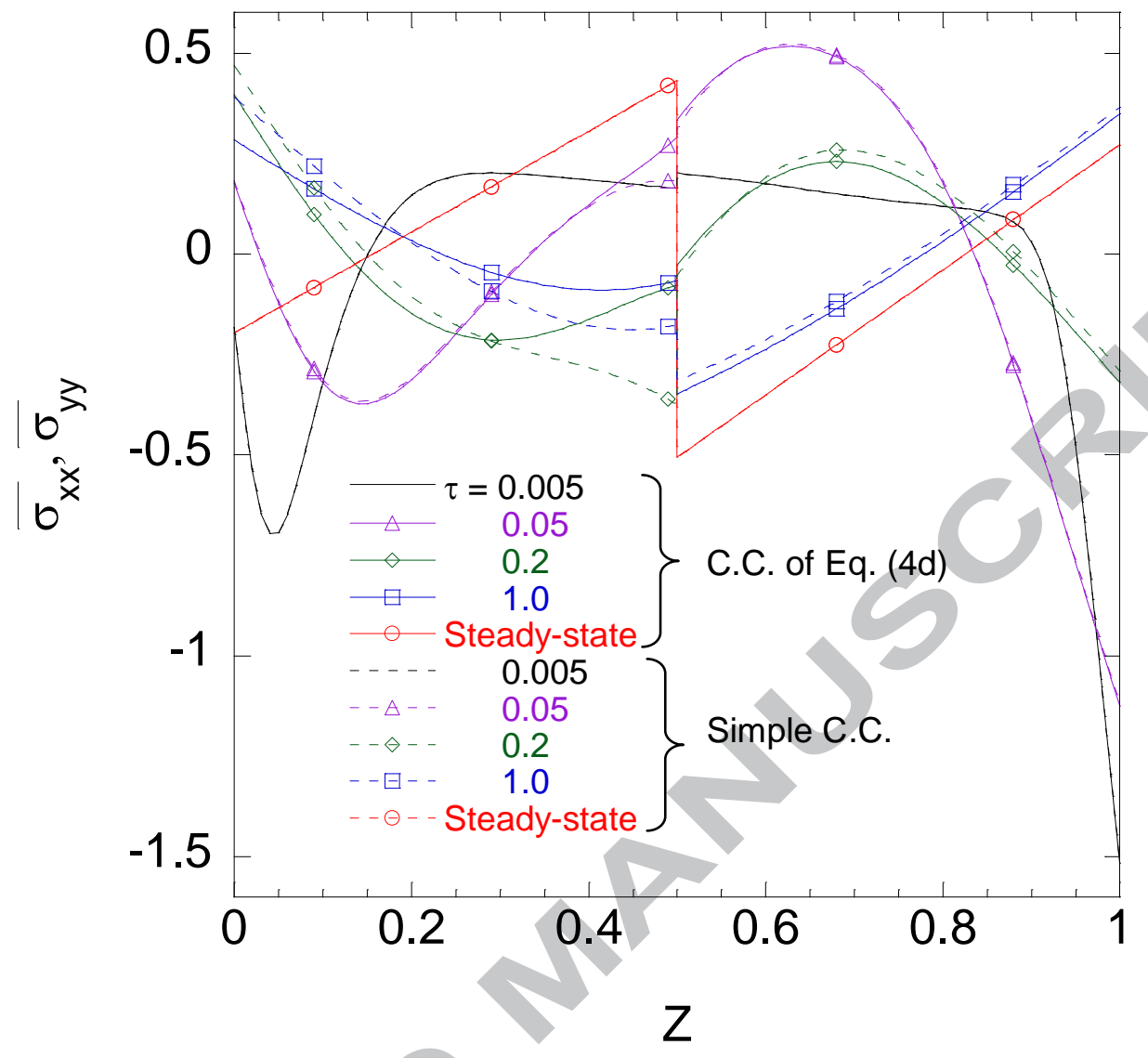

Fig. 2 


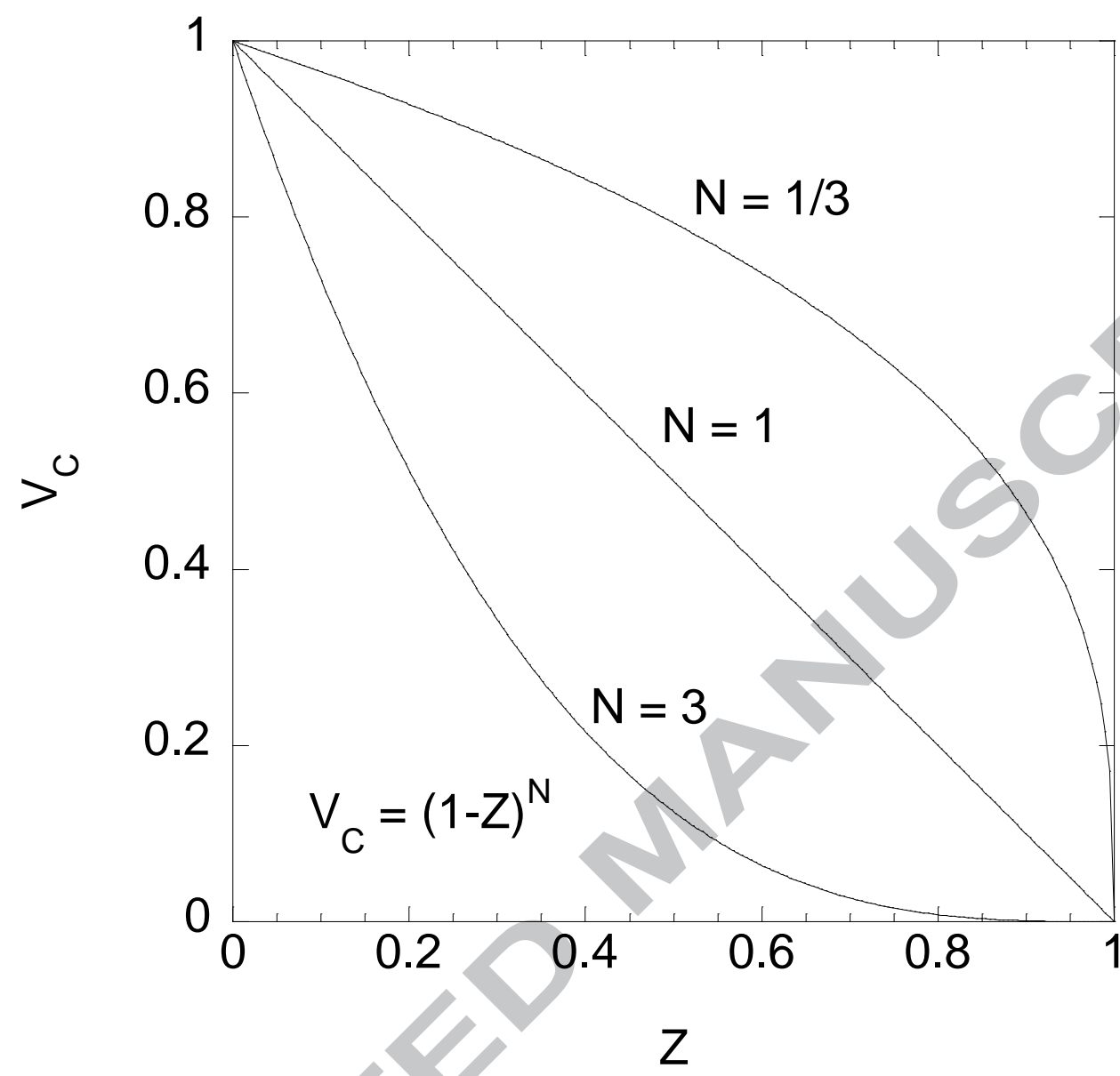

Fig. 3 


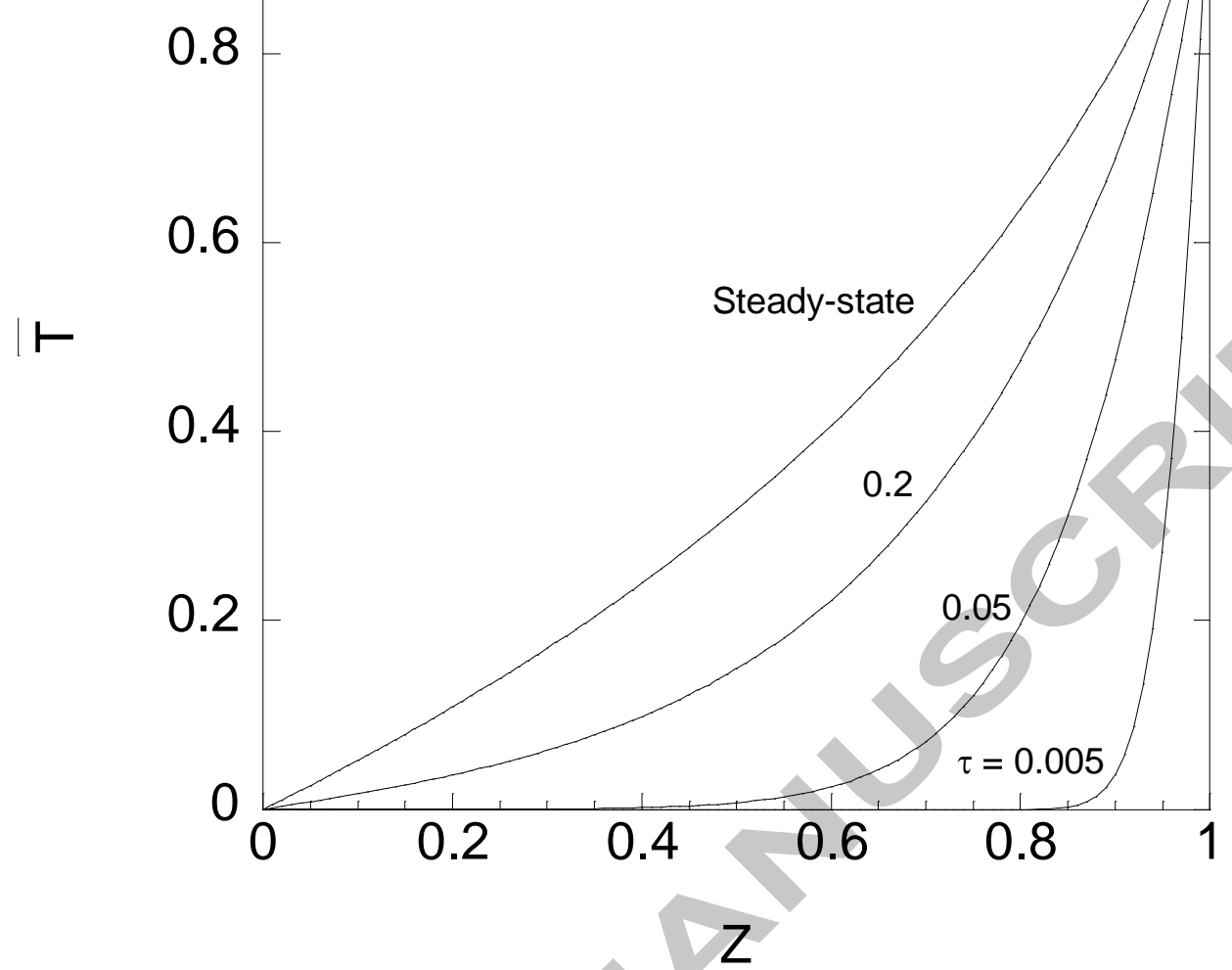

(a)

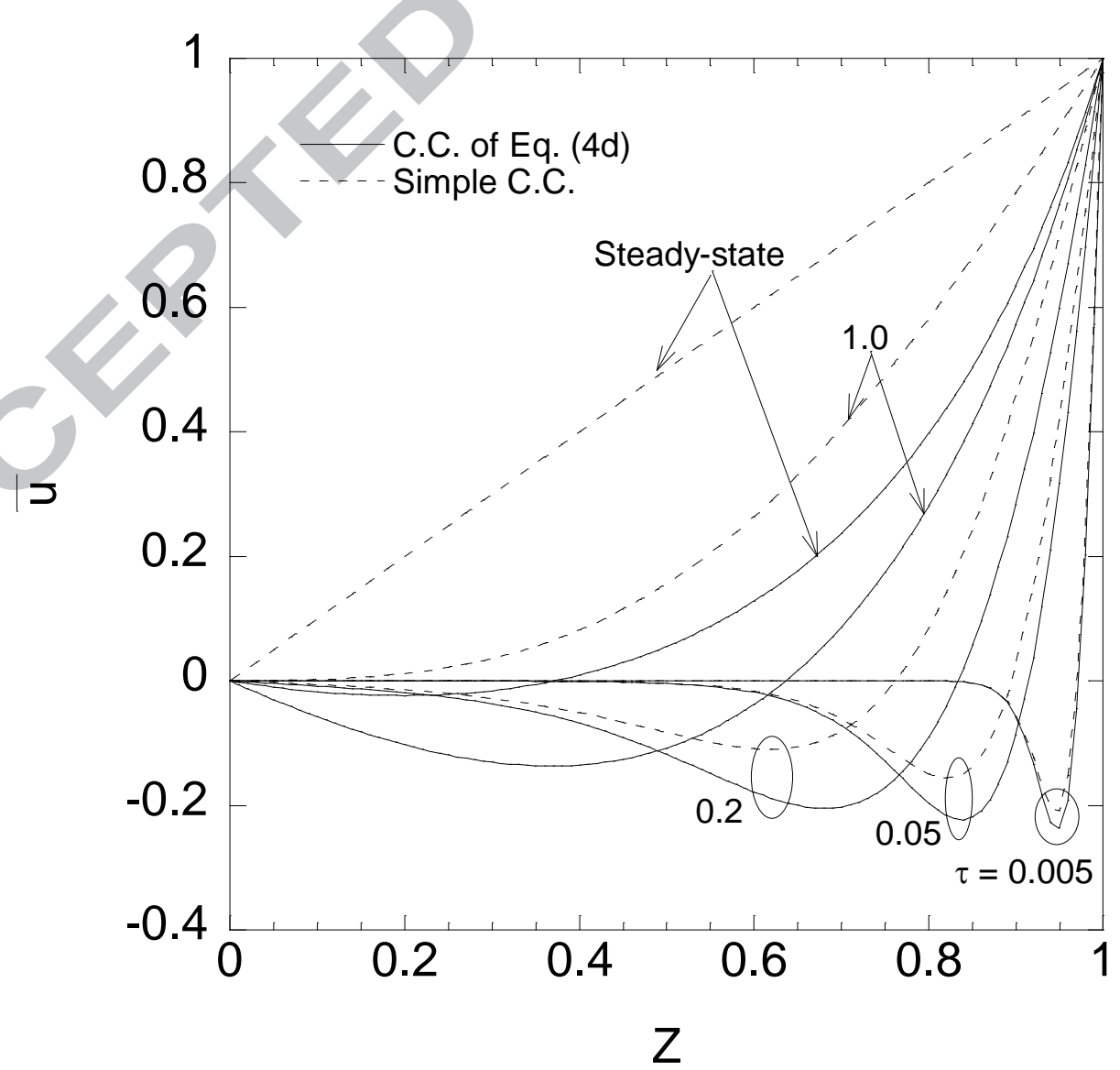

(b) 


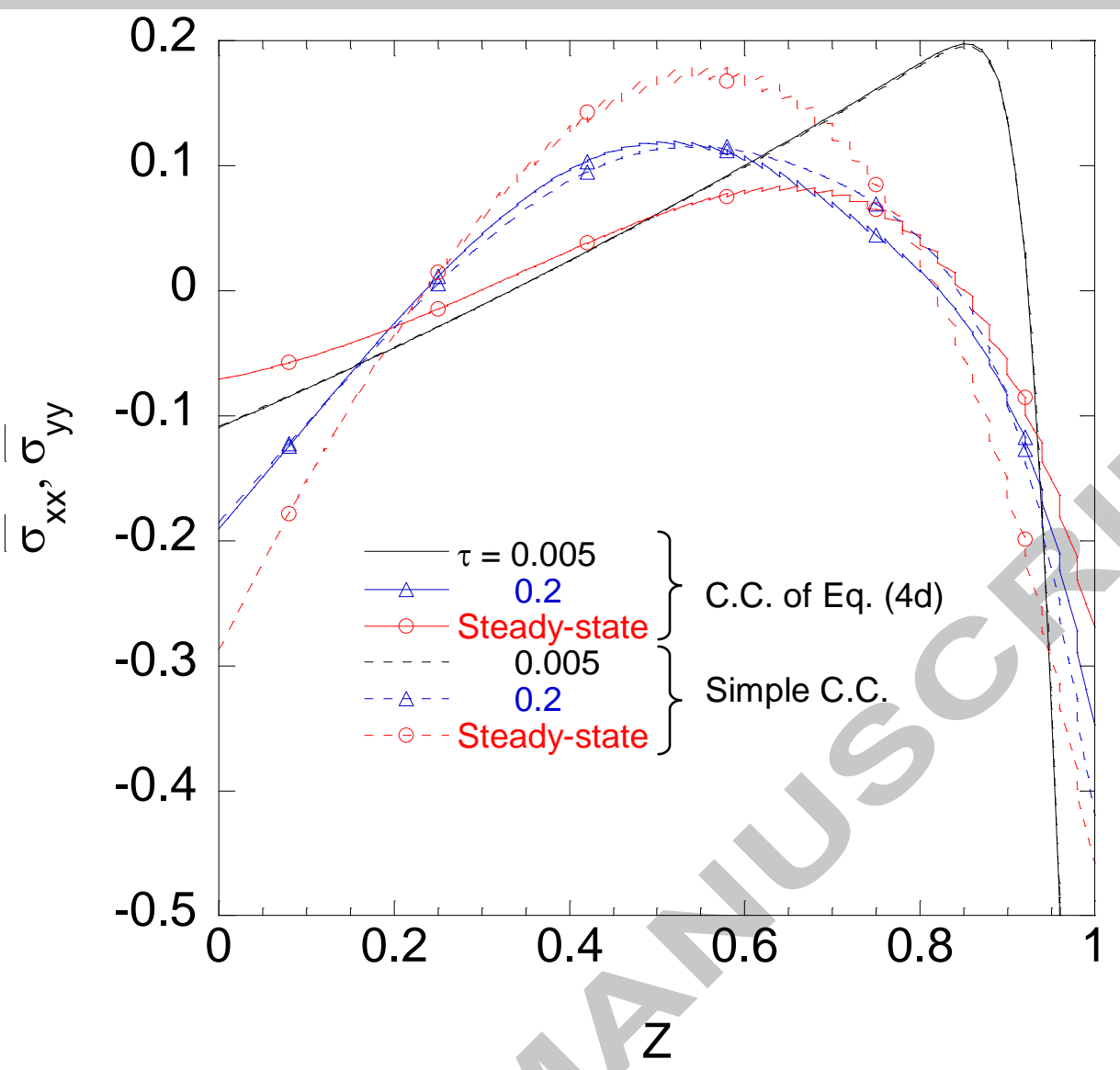

(c)

Fig. 4 


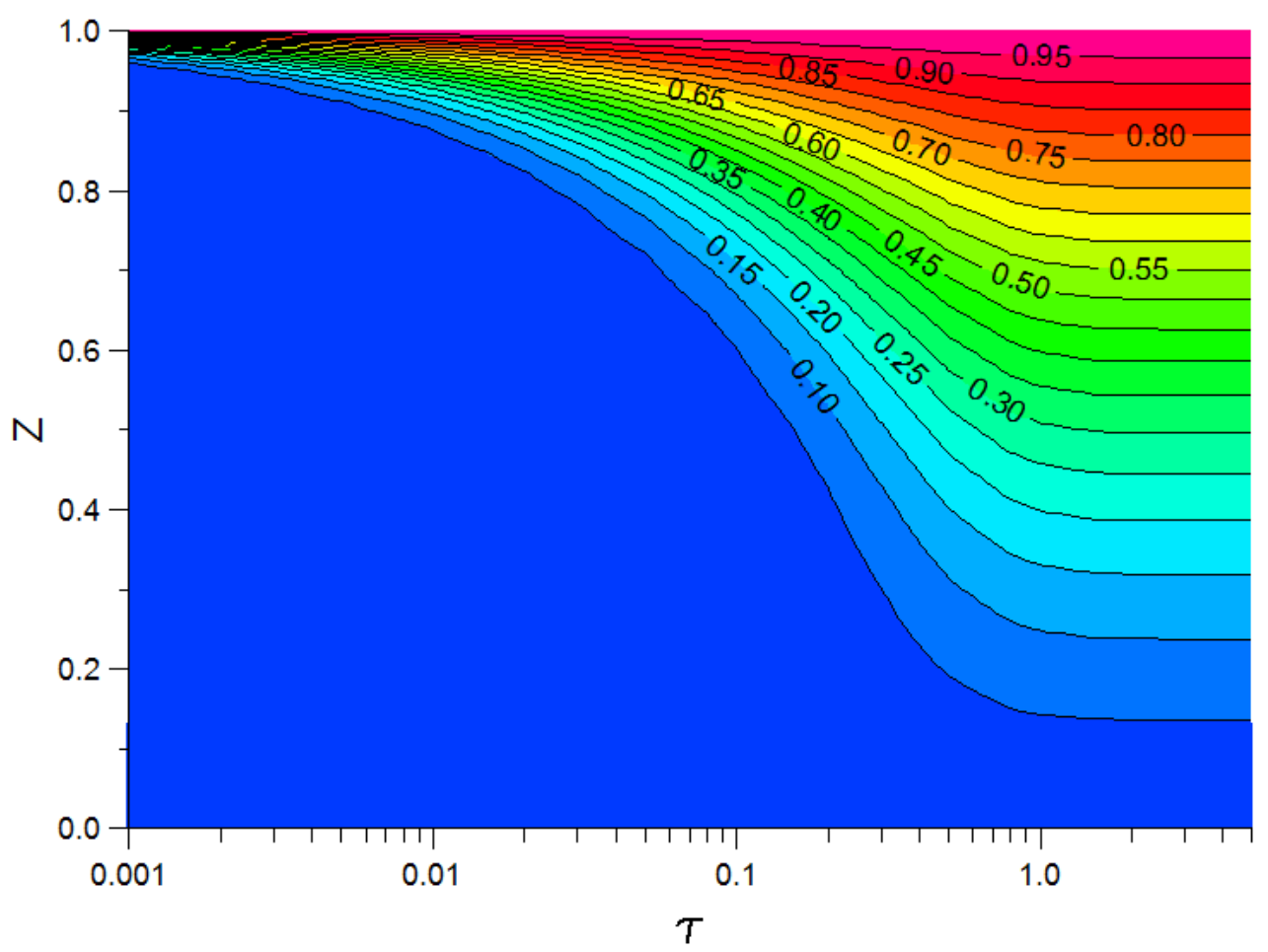

(a)

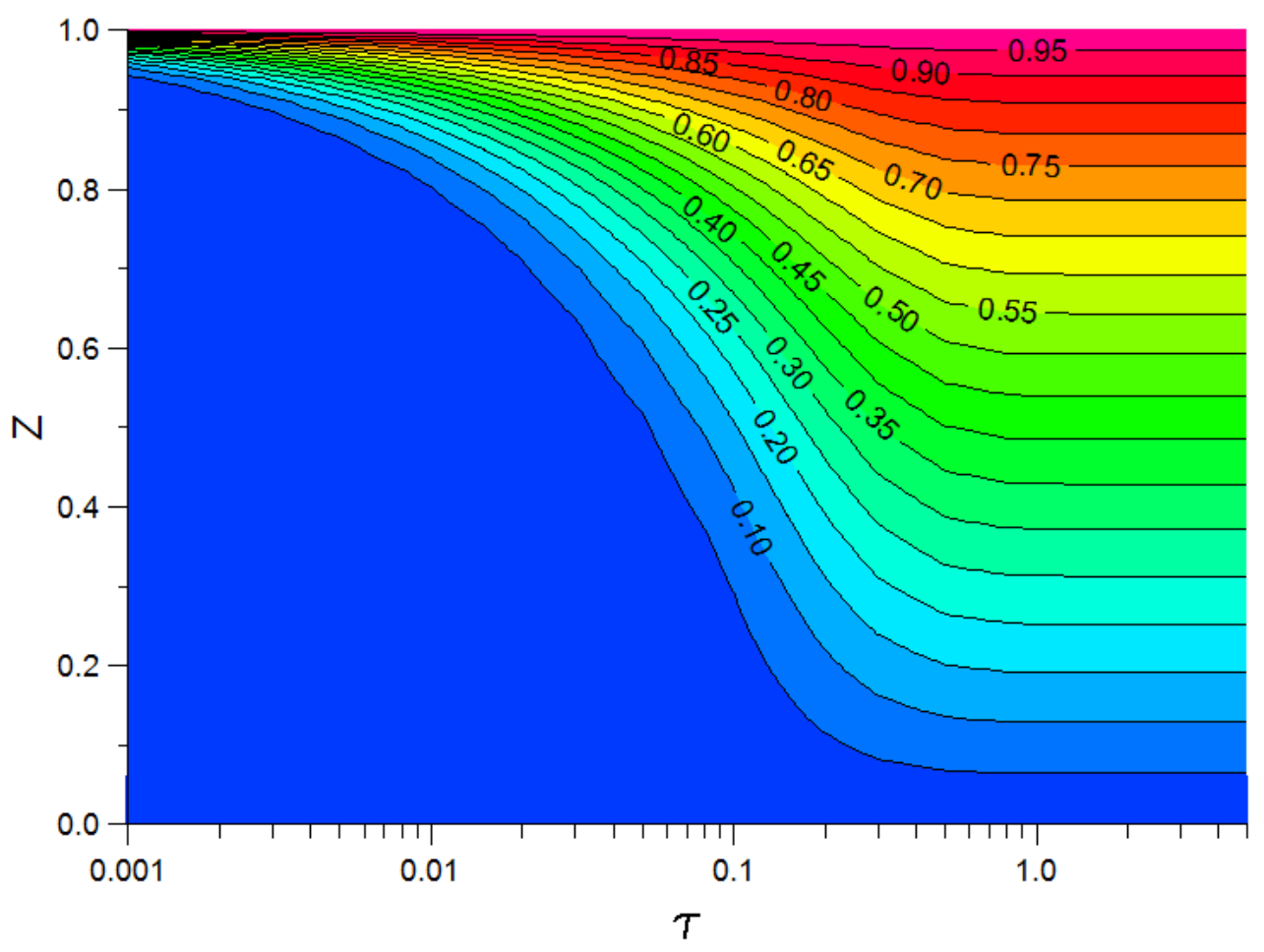

(b) 


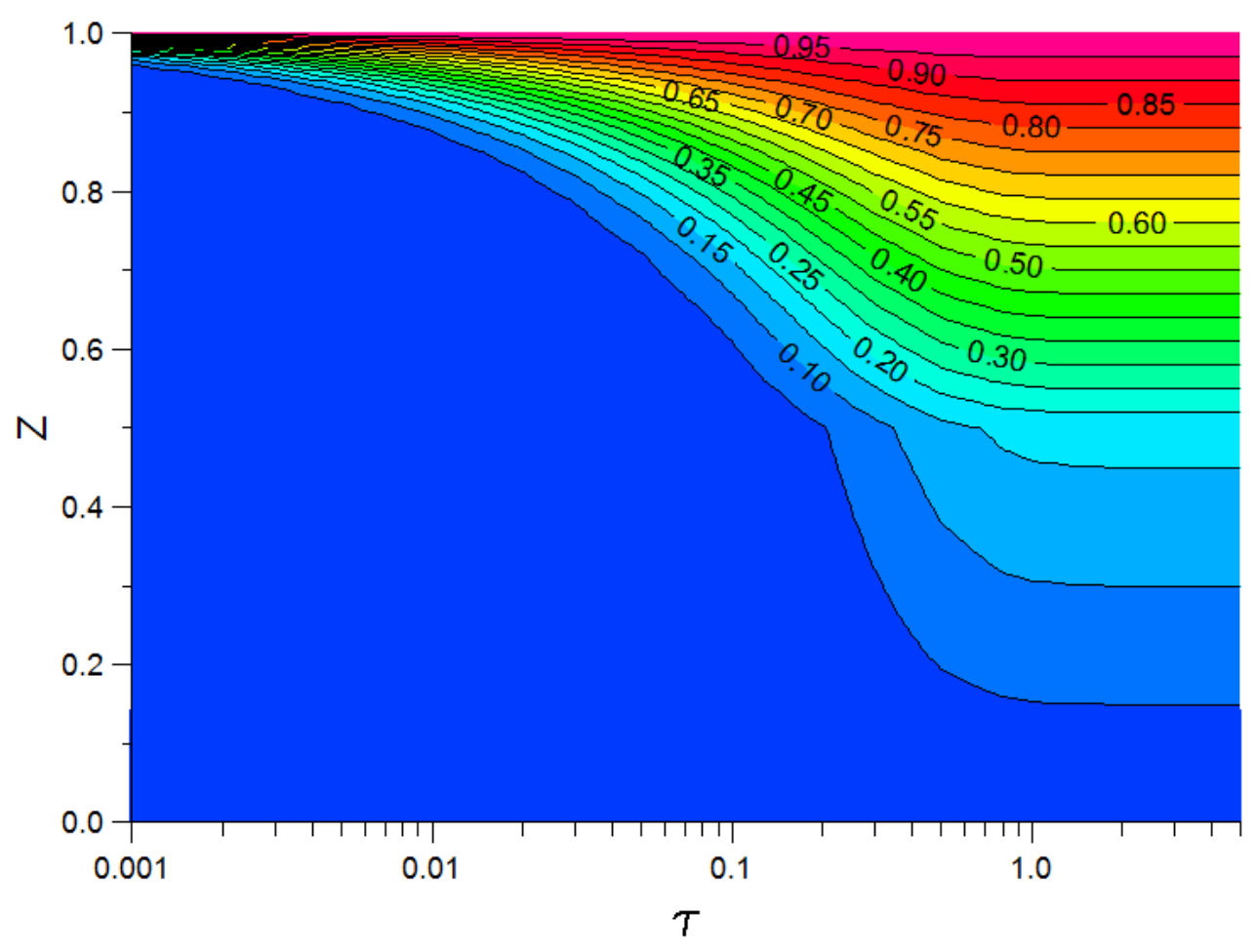

(c)

Fig. 5 


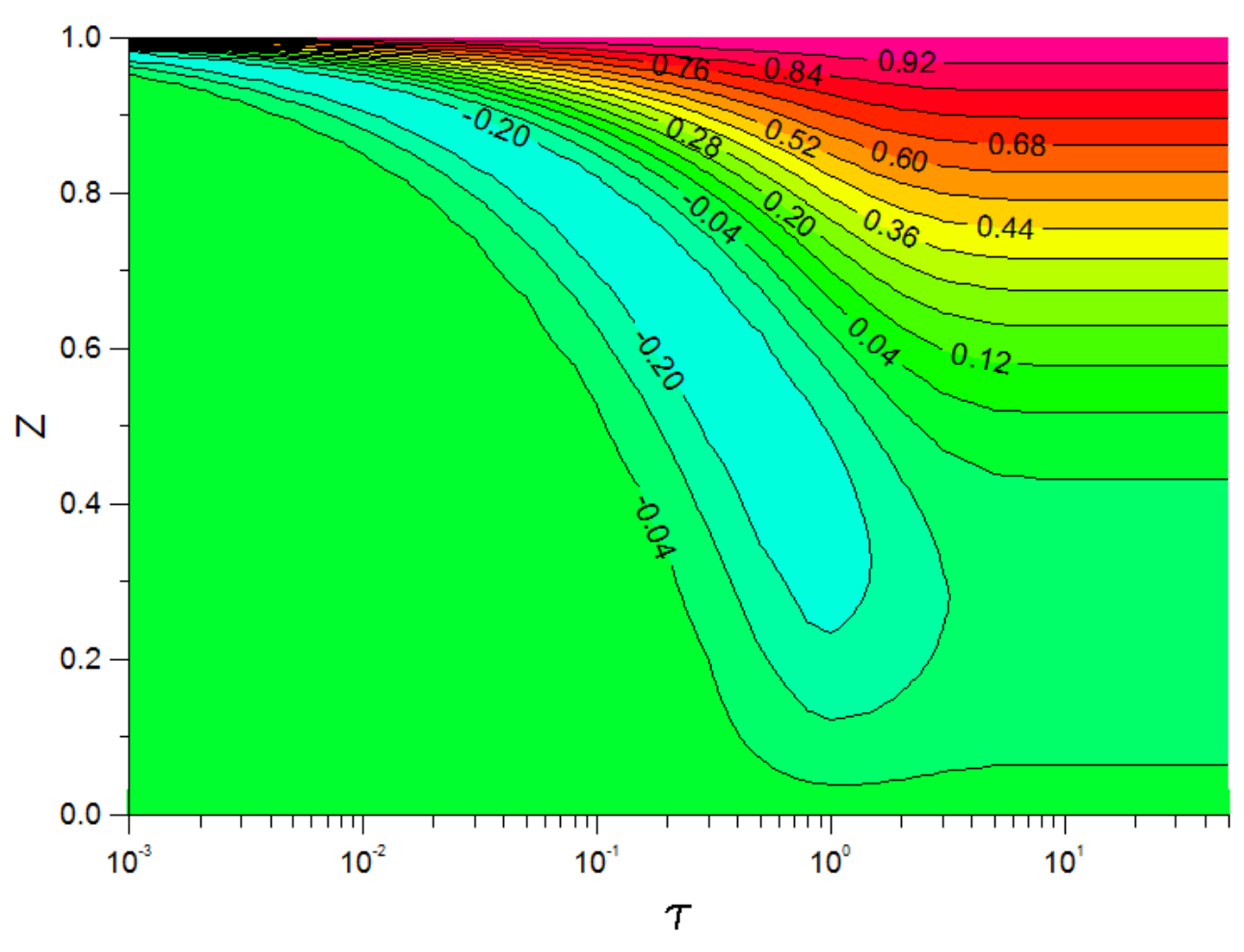

(a)

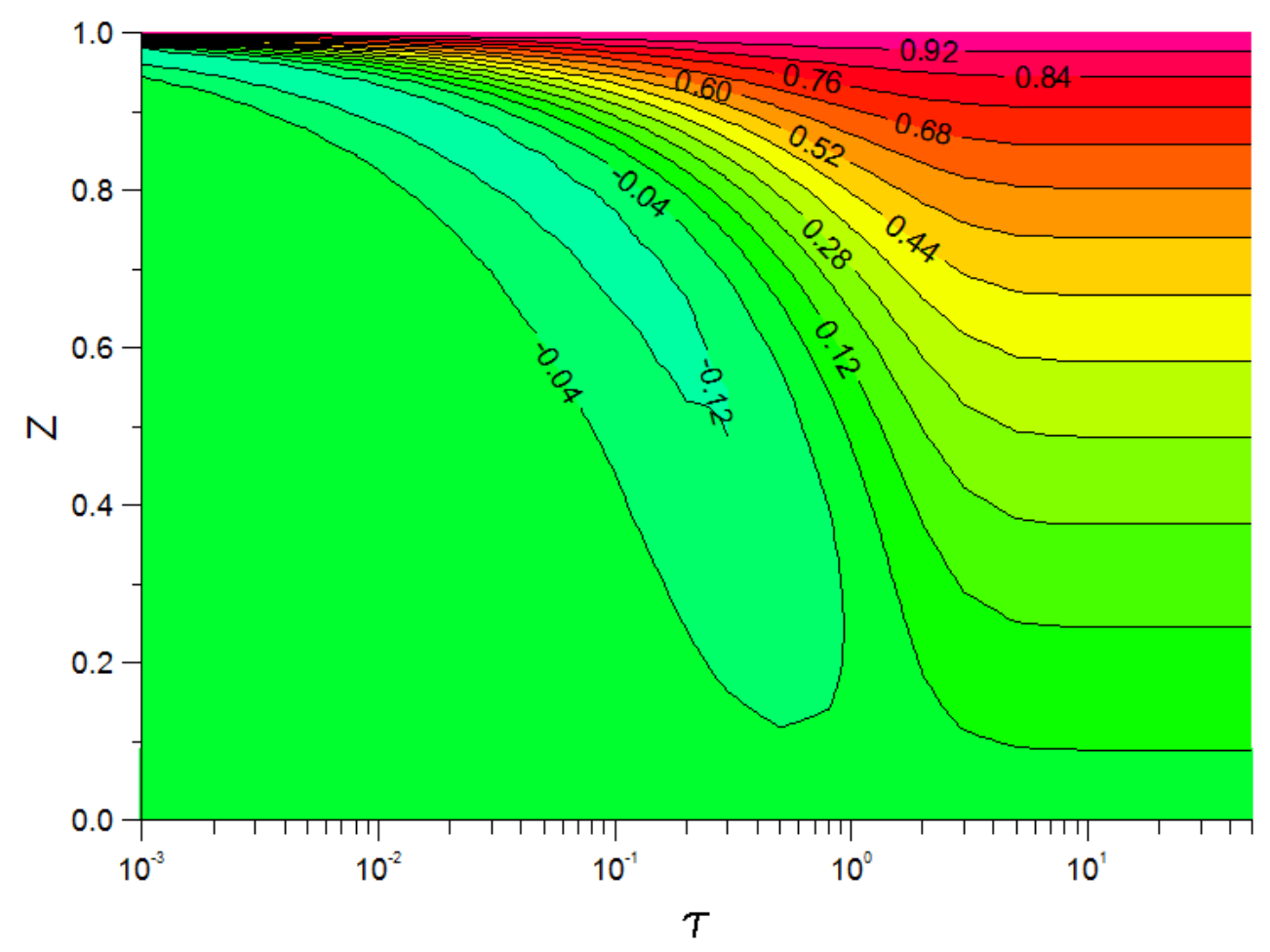

(b) 


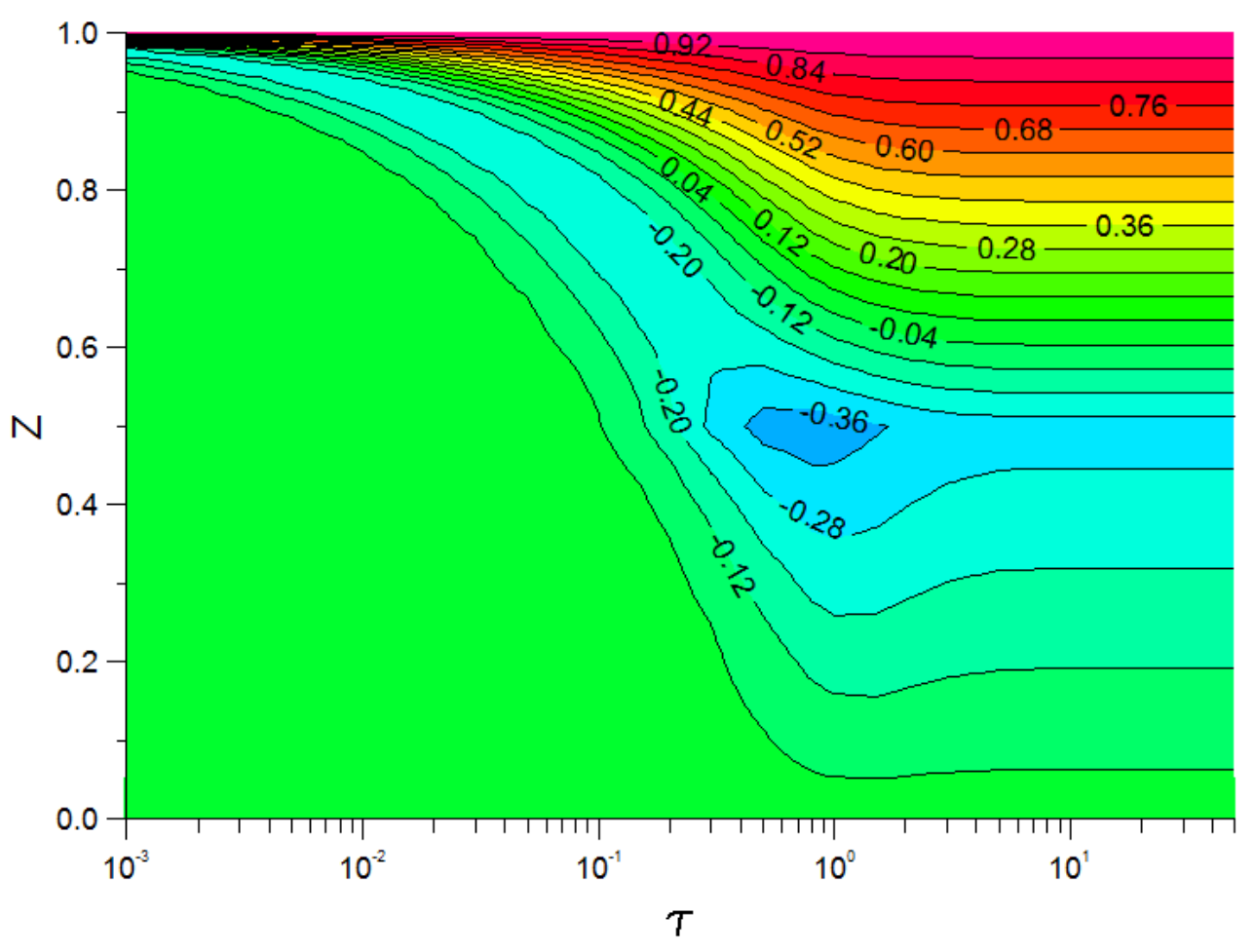

(c)

Fig. 6 


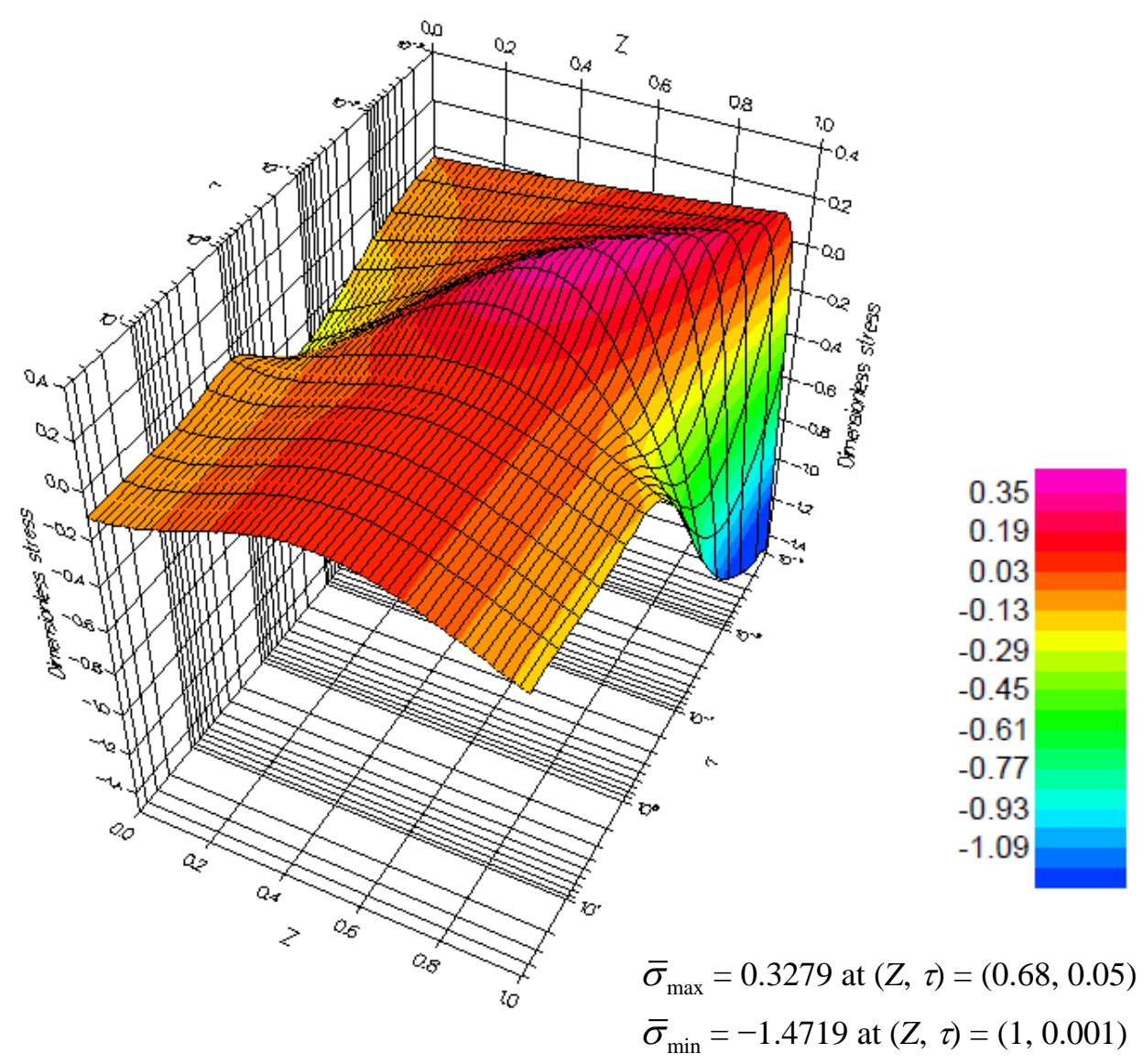

(a)

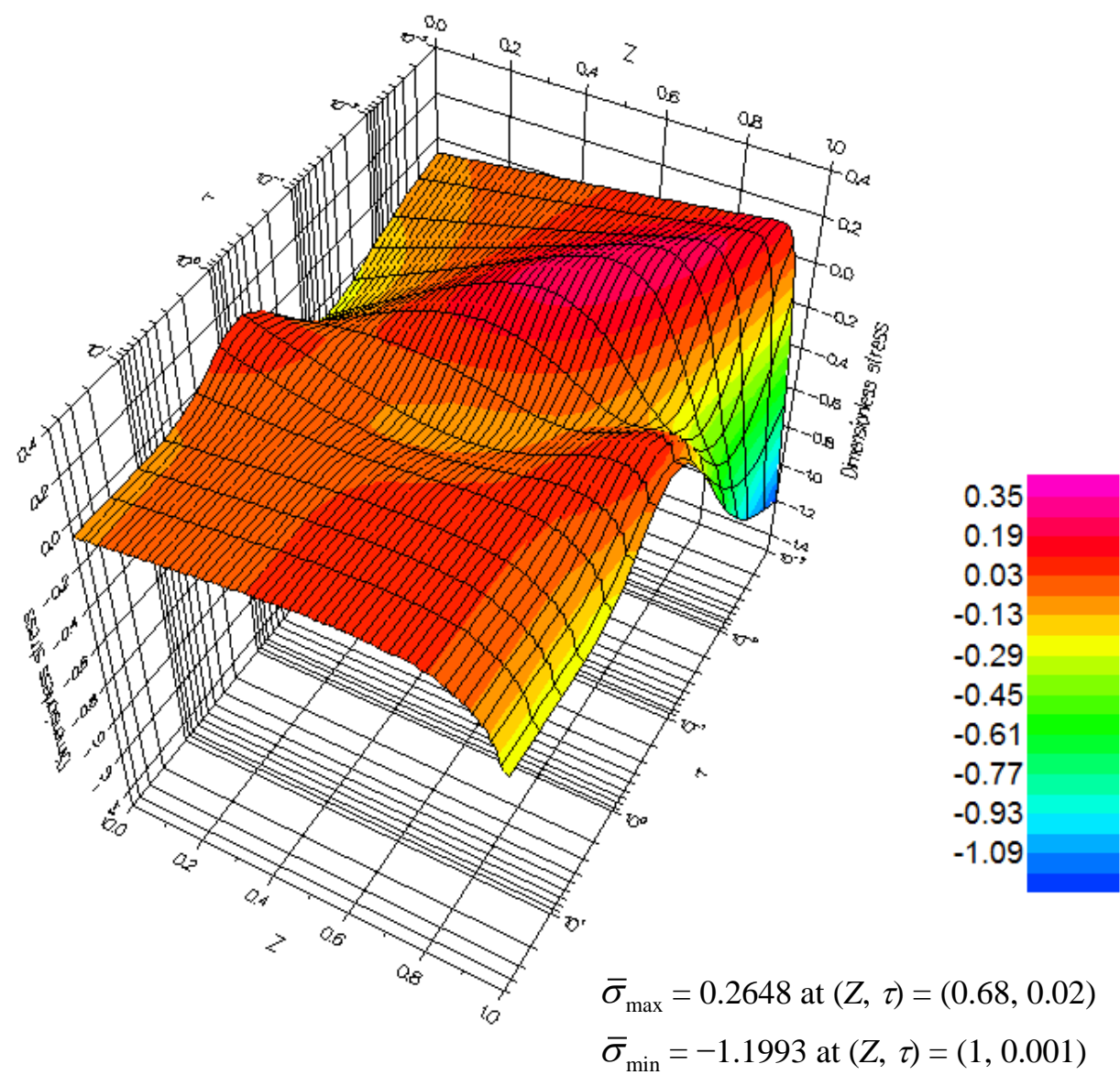

(b) 


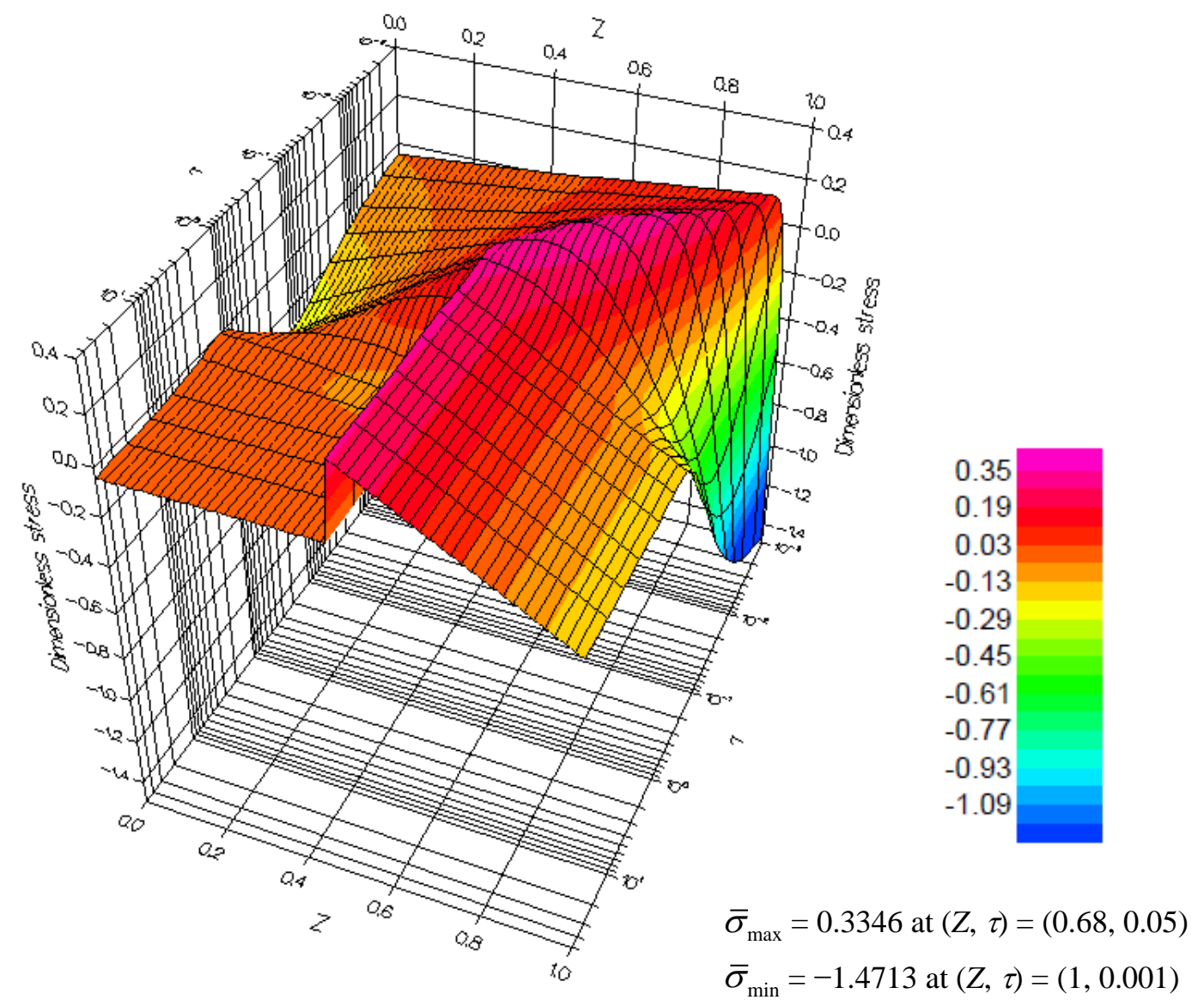

(c)

Fig. 7 